\title{
Diagnostics and Therapy for Malignant (Degenerate) Colon Endometriosis - Three Case Reports
}

\section{Zur Diagnostik und Therapie maligne entarteter Darmendometriose - 3 Fallberichte}

Authors

Affiliations

\author{
R. Schutz ${ }^{1}$, J. Woziwodzki² ${ }^{2}$ K.-W. Schweppe
}

${ }^{1}$ Endometriosezentrum Ammerland, Frauenklinik, Ammerland-Klinik GmbH, Akademisches Lehrkrankenhaus der Medizinischen Hochschule Hannover, Westerstede

2 Pathologisches Institut Aurich/Westerstede, Westerstede

\section{Key words}

- bowel endometriosis

- malignant degeneration

- endometriosis-associated carcinoma

Schlüsselwörter

- Darmendometriose

- maligne Entartung

- Endometriose-assoziiertes Karzinom

Deutsche Version unter: www.thieme-connect.de/ ejournals/gebfra

\section{received 6.8.2015 \\ revised 9.11 .2015 \\ accepted 17.11.2015}

\section{Bibliography}

Dol http://dx.doi.org/

10.1055/s-0041-109769

Geburtsh Frauenheilk 2016; 76:

417-422 (c) Georg Thieme

Verlag KG Stuttgart · New York . ISSN 0016-5751

\section{Correspondence}

\section{Prof. Karl-Werner Schweppe}

Endometriosezentrum

Ammerland

Frauenklinik

Ammerland-Klinik GmbH

Akademisches Lehrkrankenhaus

der Medizinischen Hochschule

Hannover

Lange Straße 38

26655 Westerstede

kwschweppe@ewetel.net

\section{Abstract \\ $\nabla$}

Malignant degeneration of colon endometriosis is a very rare event. We report here on three cases. A 48-year-old woman with a 10 -year history of endometriosis was treated for a rectal adenocarcinoma, a 61-year-old G1P1, who was operated at the age of 40 years for ovarian endometriosis and again at the age of 53 years for an endometriosisassociated endometroid ovarian carcinoma, presented for therapy for a lymph node recurrence of the ovarian cancer and, secondly, due to a malignantly degenerated rectum-sigmoid colon endometriosis; furthermore a 54-year old woman with a 21-year history of endometriosis was operated for malignant colon endometriosis. The tumour occurred during an adjuvant anti-oestrogen treatment with an aromatase inhibitor following surgical and radiotherapy for breast cancer. In all cases a radical cancer operation was followed by adjuvant chemotherapy and in one case with an additional radiotherapy. In the follow-up periods of 18 months, 2 and 5 years, respectively, all women remained free of recurrences. Although this is not a randomised controlled study due to the rare occurrence of such cases, a radical operation followed by individualised adjuvant therapy appears to be the treatment of choice.

\section{Introduction}

$\nabla$

Endometriosis is an oestrogen-dependent, proliferative disease that affects ca. 4 to $8 \%$ of all women in child-bearing age. It is in principle a benign disease; however, it does exhibit the properties of progression, neoangiogenesis, invasion and organ destruction that also characterise malignant processes. Sampson described for the first time the malignant degeneration of peritoneal endome-

\section{Zusammenfassung \\ $\nabla$}

Die maligne Entartung einer Darmendometriose ist sehr selten. Wir berichten über 3 Fälle. Eine 48-jährige Patientin mit 10-jähriger Endometrioseanamnese wurde wegen eines Adenokarzinom des Rektums behandelt; eine 61-jährige G1P1, die erstmals im Alter von 40 Jahren wegen Ovarialendometriose und dann im Alter von 53 Jahren wegen eines Endometriose-assoziierten endometrioiden Ovrialkarzinoms operiert wurde, kam wegen Lymphknotenrezidiv des Ovarialkarzinoms und zweitens wegen einer maligne entarteten Rektum-Sigma-Endometriose zur Therapie; ferner wurde eine 54-jährige Patientin, die seit 21 Jahren an Endometriose litt, wegen maligner Darmendometriose operiert. Der Tumor war während einer adjuvanten Antiöstrogenbehandlung mit einem Aromatasehemmer nach operiertem und bestrahltem Mammakarzinom entstanden. In allen Fällen erfolgte eine radikale Karzinomoperation gefolgt von adjuvanter Chemotherapie und in einem Fall zusätzlich adjuvanter Radiatio. Im Nachuntersuchungszeitraum von 18 Monaten, 2 bzw. 5 Jahren sind alle Frauen bisher rezidivfrei. Obwohl es keine randomisierten, kontrollierten Studien wegen der Seltenheit der Fälle gibt, scheint die radikale Operation gefolgt von einer individualisierten adjuvanten Therapie die Behandlung der Wahl.

triosis [1] and ovarian endometriosis [2], and also formulated the criteria that are still valid today to prove histologically the malignant degeneration of endometriosis:

1. both carcinomatous and benign endometrial tissue must be detectable in the same organ,

2. cancer structures and benign endometrial tissue must be histologically correlated like adenocarcinoma of the uterus is to the endometrium, 
Table 1 Immunohistochemical findings in the 3 cases.

\begin{tabular}{|c|c|c|c|c|c|c|c|c|c|}
\hline IHC & Tissue & CK7 & CK20 & ER & PR & PAX8 & WT1 & CD10 & P53 \\
\hline \multirow[t]{2}{*}{ Case 1} & benign endometriosis & + & - & + & + & + & - & + & - \\
\hline & malignant endometriosis & + & - & + & - & + & - & + & - \\
\hline \multirow[t]{2}{*}{ Case 2} & benign endometriosis & + & - & + & + & + & - & + & - \\
\hline & malignant endometriosis & + & $(+)$ & $(+)$ & - & + & - & + & - \\
\hline \multirow[t]{3}{*}{ Case 3} & benign endometriosis & + & - & + & + & + & - & + & - \\
\hline & borderline components & + & - & + & - & + & + & + & - \\
\hline & malignant endometriosis & + & $(+)$ & - & - & + & + & - & + \\
\hline
\end{tabular}

$+=$ positive, $(+)=$ weakly positive, $-=$ negative, IHC = immunohistochemistry, $\mathrm{ER}=$ oestrogen receptor, $\mathrm{PR}=$ progesterone receptor, $\mathrm{CK} 7=$ immunohistochemical marker for epithelial tumours, CK20 = immunohistochemical marker for intestinal tumours, PAX-8 = immunohistochemical marker for Müller's epithelium, WT1 = immunohistochemial marker for serous tumours, CD10 = immunohistochemical marker for endometrial stroma, P53 = immunohistochemical marker for high-grade endometrial cancer

3. the adenocarcinoma has genuinely arisen in the organ.

Corner and co-workers [3] additionally required histological evidence for a gradual transition from benign to malignant structures.

Beside the genital manifestations, an extragenital endometrial attack is found in up to $30 \%$ of the cases. These are above all colon and bladder endometrioses whereas other locations such as lungs, lymph nodes, skin etc. are very rare. With a risk of up to ca. $1 \%$ (see [4]) the malignant degeneration of an extragenital endometriosis is a rare event.

We report on 3 cases of malignant rectum-sigmoid colon endometriosis and discuss the clinical problems on the basis of literature reports.

\section{Case Reports}

$\nabla$

Case 1

A 48-year-old woman with an external diagnosis of a sigmoid colon carcinoma was referred to the surgical department for operative management. On account of dysmenorrhoea, hypomenorrhoea and uterus myomatosus, surgery involved myoma enucleation, adhesiolysis, and right-sided adnexectomy since an ovarian endometriosis was diagnosed intraoperatively. A subsequent endocrine therapy was not initiated.

In 2007 her general practitioner prescribed a gastroenterological examination to clarify perimenstrual pain and stool irregularities with slime and blood mixed in the stool. Colonoscopy revealed at $20 \mathrm{~cm}$ from the anus onwards polypoid protruding mucous membranes with a slight restriction of the lumen, tissue samples taken here exhibited a regularly formed colon mucosa. An MRI scan of the pelvis revealed a left-sided cystic ovarian lesion together with small sigmoid lesions, and an intramural contrast behaviour suggestive for endometriosis.

In 2010 a laparoscopic supracervical hysterectomy with leftsided adnexectomy and extensive adhesiolysis was performed. No information is available about the suspicion of endometriosis from the previously performed MRI scan. Histology revealed multiple leiomyomas, internal adenomyosis, regressing endometriosis cysts and a haemorrhagic corpus luteum cyst on the left ovary.

The patient was 44 years old at this time and received no further gynaecological therapy and no hormone substitution after surgical castration. With adipositas per magna (BMI 35) there were hardly any complaints about menopausal symptoms.

In June 2013 diagnostic work-up initiated by her general practitioner due to bloody diarrhoea led to the histological diagnosis of sigmoid colon cancer. Sonographic and radiological staging examinations did not provide any indications for metastasis. The tumour markers CEA and CA $19-9$ were elevated at $27 \mathrm{ng} / \mathrm{mL}$ and $600 \mathrm{U} / \mathrm{mL}$, respectively. On the gynaecological examination a cystic, partly echo-poor, partly echo-rich, poorly delineated $65 \times 37 \times 40 \mathrm{~mm}$ resistance with internal structures was found behind and above the cervical stump, giving rise to the sonographic and palpatory suspicion of recurring endometriosis of the posterior compartment including the rectovaginal septum.

An interdisciplinary re-re-laparotomy revealed after extensive adhesiolysis the tumour at the level of the retrosigmoid junction as well as further parietal tumour elements attached to the rectum. Since the frozen section analysis showed evidence for an adenocarcinoma, a rectum resection with end-to-end anastomosis was performed with the tumour being removed in toto together with the in conglomerate clogged cervix uteri. This was followed by lymphadenectomy. In the final histology, which was confirmed by an independent pathologist, pronounced endometriosis was found in the intestinal wall reaching through to the muscularis propria and submucosa. 18 of 46 regional lymph nodes had been attached by metastases, oral and aboral anastomosis rings were tumour-free, as was the cervix uteri.

On consideration of the immunohistochemical characteristics ( $\bullet$ Fig. 1 and Table 1 ) of the adenocarcinoma, it was classified as an endometrial carcinoma on the basis of colorectal endometriosis in the region of the left and right adnexa in the condition after ovarian endometriosis (pT2 L1 V0 pNx pM1 [LYM] R0 G2).

There were no complications in the postoperative course; the indication for adjuvant chemotherapy with carboplatin and Taxol was given. The patient received 6 cycles in the appropriate doses and intervals. In the follow-up period of to date 18 months there has been no evidence for a recurrence.

\section{Case 2}

A 61-year-old woman was admitted to hospital in 2008 due to persisting lower abdominal and back pain with the suspicion of a lower abdominal tumour. In 1987 the then 40-year-old patient underwent adhesiolysis, extirpation and management of endometriosis because of situation of the adhesions, lower abdominal pain and a 7-cm long endometrioma in the vicinity of the left ovary; subsequent endocrine therapy was not initiated. In 1993 hysterectomy, left-sided adnexectomy as well as extensive adhesiolysis were necessary due to recurrent endometriosis of the left adnexa with therapy-refractory lower abdominal pain as well as hyper- and dysmenorrhoea. Histology confirmed the deep infiltrating ovarian endometriosis together with adenomyosis uteri interna. 


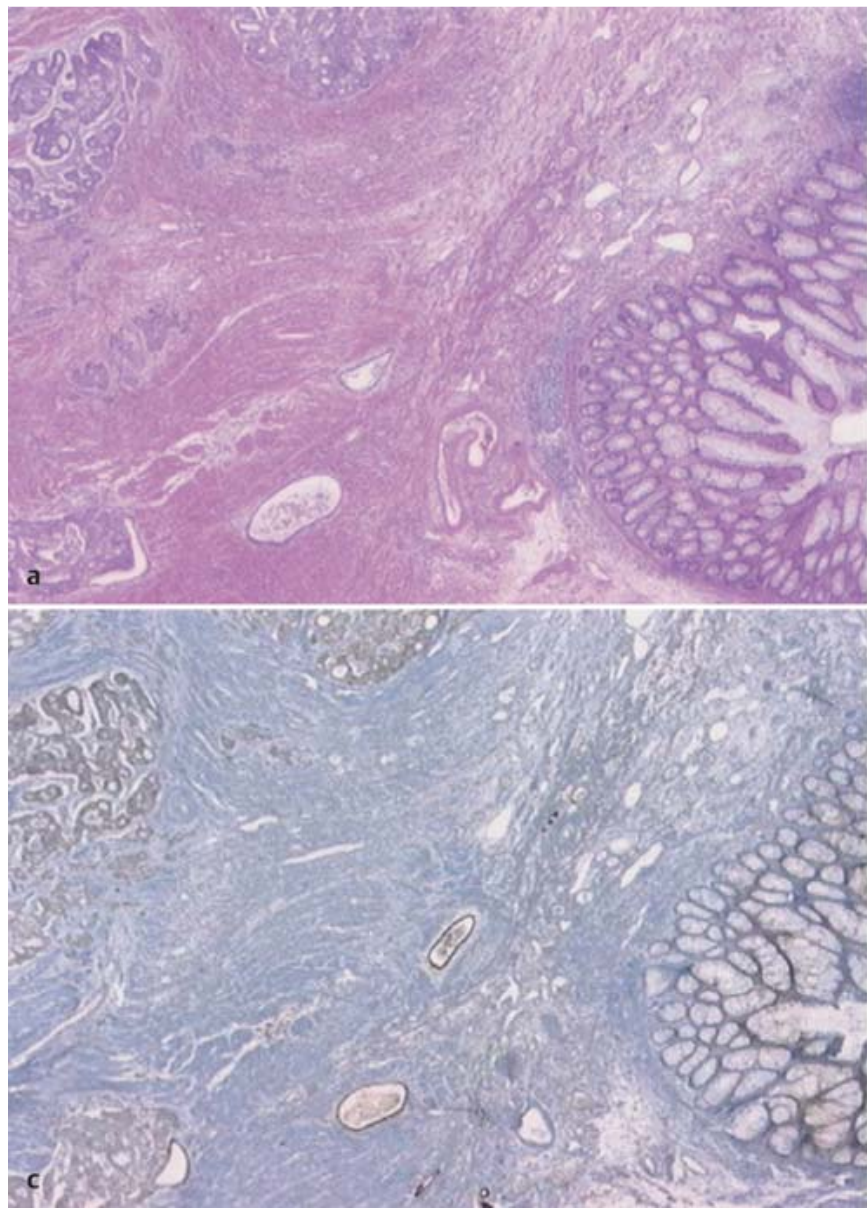

Fig. $1 \mathbf{a}$ to $\mathbf{d}$ Cross-section through the tumorous thickened intestinal wall with a slice of the lumen (case 1). One can see the normal colon mucosa (on the right of the picture) and the cancerous pockets in the intestinal wall. a Haematoxylin and eosin staining, magnification 10×. b CK7 immunohistochemical staining with positive tumour cells and negative intestinal muco-

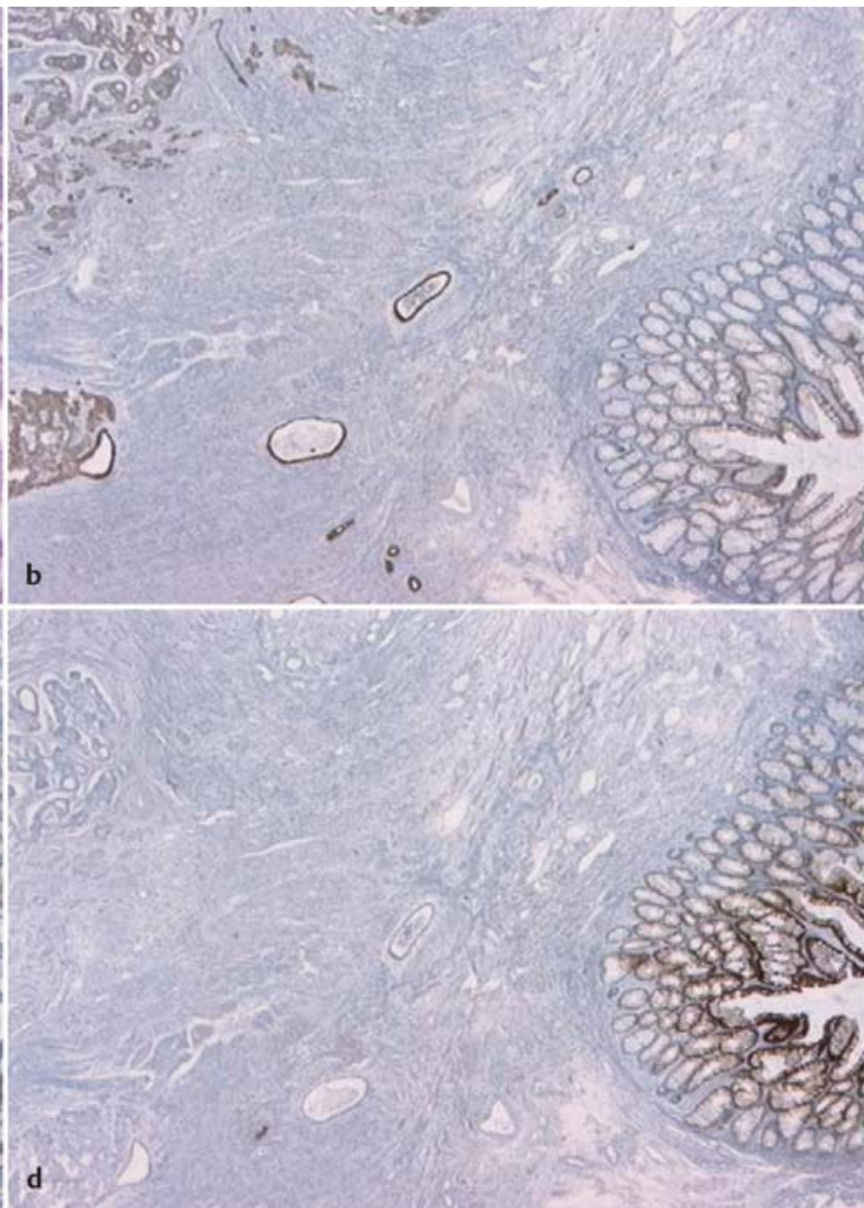

sa that excludes a primary adenocarcinoma of the intestine. $c$ Staining of the oestrogen receptors shows a weakly positive reaction of the malignant cells. d CK20 immunohistochemical staining shows negative tumour cells and positive reaction of the intestinal mucosa, in accord with the CK7 findings.
During 1997 the patient complained of moderate menopausal symptoms which, however, did not require hormone substitution therapy. In 2000 the patient underwent a renewed re-laparotomy due to right-sided lower abdominal pain and a sonographically as well as palpably unclear, right-sided, adnexa process of $10 \times 8 \times 7 \mathrm{~cm}$ in size. Histology revealed a moderately differentiated endometroid adenocarcinoma (pT1c G1-2), which was operated appropriately for its stage. The staging procedure did not reveal any lymph node metastases, or any pulmonary, bone or liver metastases. CA 12-5 with $13 \mathrm{U} / \mathrm{mL}$ was in the normal range; the tumour cells were hormone receptor positive (PR 90\%, ER $20 \%$ ). Adjuvant gestagen therapy with megestrol acetate $40 \mathrm{mg}$ daily was indicated. After about 5 years the patient terminated this therapy due to unacceptable side effects (weight gain, depressive moods).

In 2008 the patient complained again about increasing lower abdominal pain. During the diagnostic work-up a large cherrysized, poorly moveable resistance was found just above the stub of the vagina, which was not well delineated in the cranial direction, on sonography a $19 \times 18 \times 25 \mathrm{~mm}$ cystic, partly solid tumour was visualised that could not exactly be delineated from the posterior bladder wall and the anterior wall of the rectum.

A re-re-relaparatomy was performed due to the suspicion of a recurrence. After adhesiolysis palpable tumour formations were detected pararectally deep behind the stub of the vagina and in the region of the sigmoid colon. In the mesosigmoid enlarged lymph nodes were conspicuous, an intraoperative frozen section analysis revealed metastatic infiltration by an adenoid, partly papillary structured tumour that is in accord with a primary metastatic endometrial carcinoma. A deep anterior rectum resection with lymphadenectomy was performed. The final histological analysis demonstrated two types of tumour formations:

1. a poorly differentiated ER-negative, PR-negative adenocarcinoma in the vicinity of the regional lymph nodes, the mesocolon and mesorectum as well as paraaortically,

2. cystic dilated endometriosis structures with atypically transformed epithelial formations in the form of a papillary-serous carcinoma in situ with tumour propagation in the region of the intramural neural plexus of the intestinal wall and invasive tumour elements in the region of the intestinal wall endometriosis. In addition, focal dilated endometriosis cysts without atypical cell formations.

The resection margins were free of tumour. Eight of the total of 14 removed lymph nodes had been attacked by metastases.

In conclusion, the findings were classified on the one hand as endometriosis of the rectum-sigmoid colon with progressive dysplasia, carcinoma in situ and perineural invasion and, on the other hand, as lymph node recurrence of the endometrial ovarian 


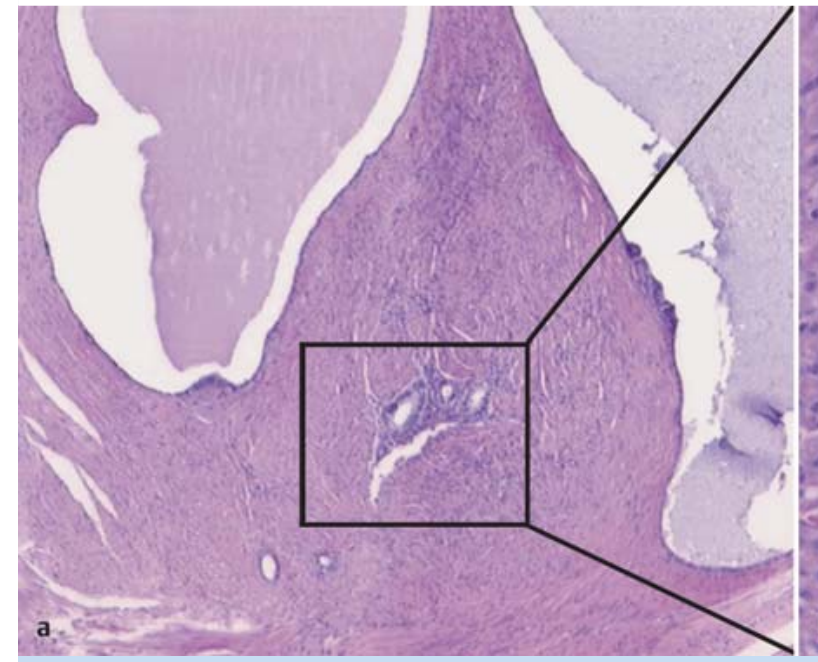

Fig. $\mathbf{2} \mathbf{a}$ and $\mathbf{b}$ Intestinal wall with benign endometriosis (case 3 ). Hyperplastic thickened intestinal wall with cystic dilated endometriosis glands (a), filled with secretions with flattened, inactive epithelium. In addition, islands with

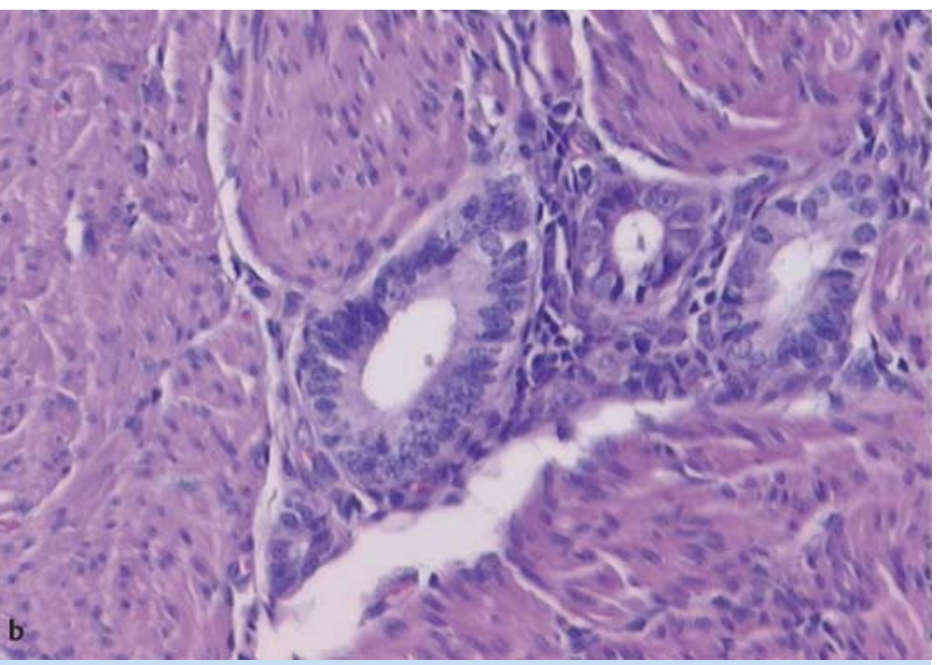

proliferating endometriosis surrounded by fibrosis and muscle cells (b). staining HE; magn. $\mathbf{a}=10 \times$ and $\mathbf{b}=100 \times$.

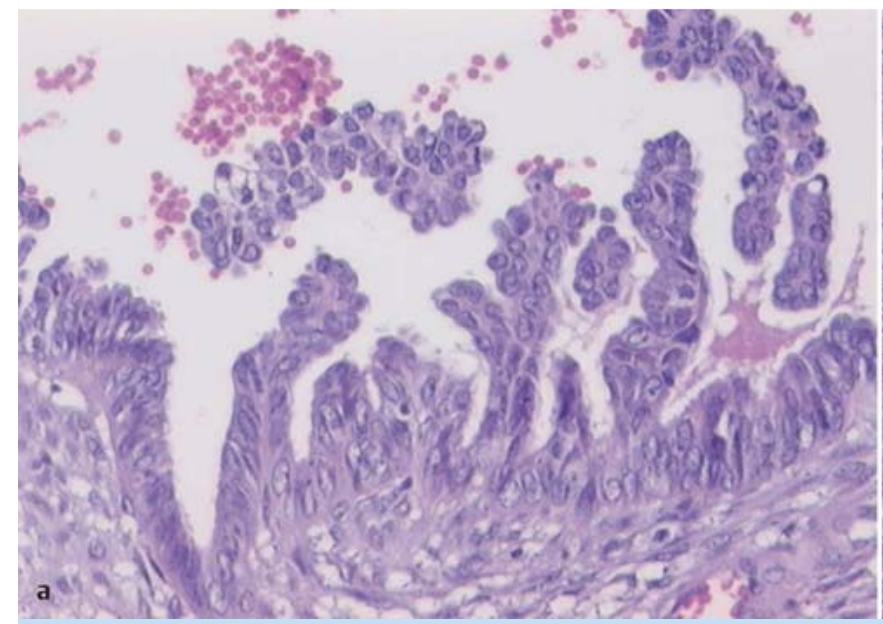

Fig. $\mathbf{3} \mathbf{a}$ and $\mathbf{b}$ Varying differentiation of the malignancy. The dedifferentiation spectrum of the tumour in case 3 , classified as $G 2$, ranges from borderline parts (a) with epithelial high-grade atypical cell conglomerates without

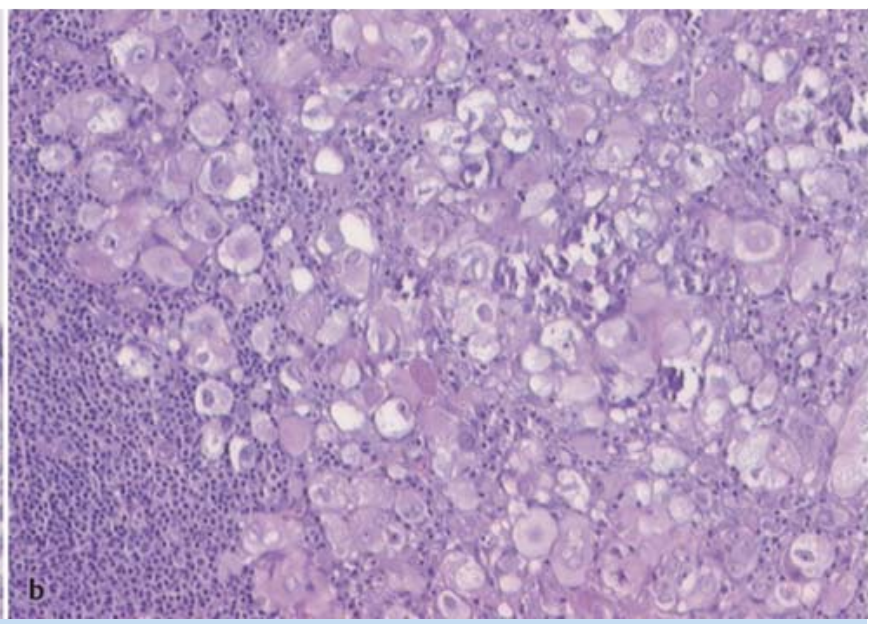

detectable invasion through to little differentiated carcinoma cells in the lymph node metastases (b). carcinoma treated surgical and with adjuvant therapy 8 years previously. The patient received an adjuvant chemotherapy with carboplatin AUC5 and Taxol $\left(175 \mathrm{mg} / \mathrm{m}^{2}\right)$ in the appropriate doses and intervals. All follow-up examinations of the abdomen were unremarkable. A second recurrent disease had not occurred at five years after surgery.

It should be mentioned that two years ago the patient underwent breast-conserving surgery and received adjuvant therapy for a poorly differentiated invasive ductal breast cancer (pT1c pTIS L0 V0 pN0 [SN 0-1] R0). The patient has also not suffered from a recurrence of the breast disease.

\section{Case 3}

A 54-year-old woman was admitted to hospital in 2009 because of recurring abdominal pain, constipation, loss of appetite and loss of energy with an unclarified tumour in the lesser pelvis. 21 years before, the then 33-year-old woman underwent a hysterectomy and resection of a deeply infiltrating parametric and retro- vaginal septum endometriosis by laparotomy on account of recurrent, therapy-resistant hypermenorrhoea and dysmenorrhoea. A postoperative endocrine therapy was not indicated. In 1993 a continuous gestagen therapy with medrogestone $5 \mathrm{mg}$ daily was started because of a suspected recurrent endometriosis (cystic ovarian endometriosis). This was stopped after 14 months when the patient was diagnosed with a left-sided invasive ductal breast cancer (pT2 pN0 [SN] M0 G3, ER 40\%, PR 30\% Her2/neupositive). In 1995 a breast-conserving operation with radiotherapy and adjuvant chemotherapy was carried out (4 cycles of EC scheme followed by an antioestrogen therapy with anastrozole $1 \mathrm{mg} / \mathrm{d}$ ). Furthermore an adjuvant bilateral laparoscopic adnexectomy was performed (histology of the ovaries did not show any evidence of endometriosis, merely functional cysts and serous membrane inclusion cysts).

On clinical examination and vaginal sonography, a good tabletennis ball-sized, firm elastic, immobile tumour was conspicuous above and dorsal from the vaginal stub, the rectal mucous mem- 
branes could be moved. A colonoscopy performed 7 months earlier because of constipation and lower abdominal pain did not reveal any abnormal findings. An MRI scan demonstrated a 3-cm, solid tumorous structure above the vaginal stub that could not be exactly delineated from the rectal wall.

With the suspicion of recurrent endometriosis a re-laparotomy was performed and, after difficult adhesiolysis of the frozen pelvis, a mandarin-sized tumour reaching from above and dorsolateral of the vaginal stub to the paraproctium was detected. The frozen section analysis confirmed the clinical suspicion of malignancy so that after total mesorectal mobilisation a deep anterior rectum resection with end-to-end anastomosis was performed.

The histological analysis revealed a moderately differentiated endometrial adenocarcinoma ( $32 \mathrm{~mm}$ in size) on the bed of a previously existing endometriosis ( $\boldsymbol{O}$ Fig. $\mathbf{2}$ ) in the vicinity of the rectal wall with infiltration into all layers of the wall through to the submucosa. In the vicinity of one tumour part changes were seen that corresponded to a borderline tumour. Of 26 regional lymph nodes only one had been attacked ( $\bullet$ Fig. 3). The fibrolipomatous pelvic connective tissue (residual parametria) was tumour-free, as was also the resected vaginal stub. The patient received an adjuvant therapy comprised of 6 cycles of cisplatin/doxorubicin and in sequence radiotherapy of the lesser pelvis and pelvic lymph drainage pathways until January 2010. Tumour follow-up findings including imaging procedures (sonography and MRI of the lesser pelvis) have remained unremarkable up to date.

\section{Discussion}

Malignant degeneration of endometriosis is a rare event and malignant degeneration of intestinal endometriosis is extremely rare. During the 5-year period reported here (2008-2012) we have operated on 3416 patients for endometriosis; 263 of them for rectum-sigmoid colon endometriosis (7.7\%). We observed 13 endometriosis-associated malignancies $(0.38 \%$ of all operated endometriosis cases); among them were the 3 cases described here. This corresponds to an incidence for malignant degeneration of extragenital cases of $23 \%$ of all endometriosis-associated malignancies or, respectively, 0.88 per thousand of all endometriosis cases and $1.14 \%$ of all intestinal endometrioses. Thus, our figures are lower than those given in the literature over the past few years where a risk of $2.5 \%$ for the malignant degeneration of ovarian endometriosis was calculated $(6 \times$ higher than in our collective) [5]. These differences can be explained by considering that the in part low case numbers, the nature of the publishing facility (gynaecology, surgery, pathology) and specialisation of the hospital all have a strong influence on the investigated collectives. An interdisciplinary, multicentre trial is needed to provide valid incidence data and clinically relevant risk estimations; such a trial is currently being carried out by Ulrich and co-workers [6]. According to a recent literature review [7] 80\% of all extragonadal endometriosis-associated malignancies have their origins in the rectum-sigmoid colon and on histology two thirds of the cases prove to be adenocarcinomas, $10 \%$ each endometroid stromal sarcomas and adenosarcomas as well as malignant Müller's mixed tumours in $5 \%$ of the cases. Thus, our three cases correspond to the most frequent location and the most frequent histological type.

The differential diagnosis between endometroid adenocarcinoma and primary adenocarcinoma of the colon is difficult, not only for the clinician but also for the pathologist. The symptomatics are identical, but clinically the lack of attack on the intestinal mucous membranes points to an endometroid process, ultimately, however, immunohistochemical examinations are necessary to make an exact diagnosis. A primary colon carcinoma is CK-20 positive and CK-7 negative whereas, in contrast, an endometroid carcinoma is CK-7 positive and CK-20 negative (for details of the cases, see $\odot$ Tab. 1). However, since individual tumour parts can exhibit different receptor expressions and immunohistochemical reactions (case 1 ), the differential diagnosis can be problematic. This is also apparent for case 3 where the negative response for oestrogen receptors can be considered as a sign of dedifferentiation, whereas the CD 10 negative with positive P53 result suggests for these differently differentiated carcinomas there are also areas that correspond to a highly differentiated endometroid carcinoma.

Furthermore, it is a matter of discussion if the TNM classification is meaningful for extragenital malignant endometriosis and whether it should be classified as an ovarian or an intestinal cancer. Thus, for example, this question can be posed in case 3 in which a hysterectomy had been performed in 1988, a bilateral adnexectomy in 1995 and in whom in the absence of internal genital organs a malignant colon endometriosis was operated in 2009. As a colon carcinoma the classification pT3LOV0pN1 (1/26) pMx R0 G2 would have been correct because the malignancy had originated from tissue of the genital organs while pT3 pNx pM1 (LYM + intestine) G2 would have been logical. Similarly in case 1, in whom in 2003 a myoma enucleation with right-sided adnexectomy and in 2010 a supracervical hysterectomy with left-sided adnexectomy were performed, both because of endometriosis and myomas. In 2013 a sigmoid colon resection and lymphadenectomy with resection of the cervix uteri were carried out. For an intestinal carcinoma the classification pT2L1V0pN2 (18/46) pMx R0 G2 would be correct whereas for an ovarian carcinoma pT2L1V0pNxpM1 (LYM) R0 G2 would be correct. For the surgical procedure the assignment as intestinal carcinoma is helpful whereas for the adjuvant therapy the phenotype of the carcinoma is rather more relevant. Accordingly, the locoregional lymph nodes of the colon are removed surgically, the adjuvant chemotherapy with carboplatin and Taxol then takes the metastatic (pM1 LYM) endometroid carcinoma into consideration. Since no tumour formula is appropriate for the situation of extragonadal malignant endometriosis, it is recommended to disregard the organ classification according to the TNM system and to descriptively report the pathological findings phenotype, tumour size and resection margins, lymph node attack and metastasis.

At the time of diagnosis the average age was 55.4 years with a standard deviation of 12.8 years, whereby at this time the youngest patient was 33 years old and the oldest 80 years [7]. Our cases were also peri- or, respectively, post-menopausal women and Ulrich and co-workers [6] pointed out that in cases of recurrence of endometriosis symptoms in peri- and post-menopausal patients the clinician should always consider the possibility of a malignant degeneration of the already known and documented endometriosis.

In many of the previously published cases the patients had received long-term oestrogen monotherapies, but endometriosisassociated carcinomas have also been described under Taxol or in one case, respectively, under gestagen therapy [8]. We observed carcinomas without any influence of endocrine therapy (case 1) or, respectively, after terminated gestagen therapy (case 2) and during an antioestrogen therapy with an aromatase inhib- 
itor (case 3). The latter situation had not been reported previously.

Individual case reports and small series do not allow for any clear recommendations about endocrine therapy for postmenopausal symptoms in patients with endometriosis; however, an indicated oestrogen administration should always be combined with a gestagen [9]. The pathophysiological background for this recommendation is that oestrogens not only stimulate the proliferation of oestrogen receptor-positive cells but also influence the prostaglandin metabolism by means of an increased cyclooxygenase activity. This favours a resistance to apoptosis and in relation with the hyperoestrogenism (favoured locally by aromatase activity) leads to a higher risk for malignant transformation. Various other molecular biological mechanisms for endometriosis-associated malignancies of the ovary have been discussed. Thus an $\mathrm{Fe}^{++}-$ overload in the endometriosis lesion can lead to protein changes and DNA damage through an elevation of oxygen radicals [10] and inactivation of the PTEN tumour suppressor gene as the first step for a malignant transformation [11]. It has not yet been examined if this mechanism is also applicable for the malignant degeneration of extragonadal endometriosis.

The clinical picture of extragonadal endometriosis frequently reflects its localisation [12]. Vaginal bleeding following a hysterectomy, rectal bleeding from the posterior compartment in a patient with a history of unspecific lower abdominal pain have also been reported along with stool irregularities, constipation and intestinal cramps. Thus, in the diagnostic work-up an interdisciplinary procedure has proved valuable since colonoscopy is often unremarkable, as pointed out by Yantiss and co-workers [4] in their series of 17 cases. Rectovaginal palpation, sonography and radiological imaging procedures point in the right direction but only histology is decisive.

Surgical treatment follows the principles of cancer surgery in the lesser pelvis with the target of R0 resection and regional lymphadenectomy. Chemotherapy with platinum-containing substances combined with taxanes is recommended as an adjuvant therapy [13], which was also performed in our case 1 whereas in case 3 a combination of platinum with anthracycline was administered.

On the other hand, it has been suggested that patients with, above all, extragonadal endometriosis-associated malignancies that are limited in extent to the lower pelvis, will benefit more from adjuvant pelvic radiotherapy [8]. Furthermore, a high-dose gestagen therapy may possibly have a similar effect on gestagen receptor-positive malignancies as is known from the therapy for endometroid cancer. This was the reason in our case 2 for the adjuvant high-dose gestagen therapy for the endometroid ovarian cancer stage Ic.

There are also discrepancies in the literature with regard to the prognosis. Some authors found no differences between endometriosis-associated and non-endometriosis-associated malignancies $[14,15]$ while others found a better prognosis of EAM [8, 16]. The relatively favourable prognosis for endometriosis-associated malignancies in comparison to primary ovarian cancer is explained, on the one hand, as a result of genetic mutations of endometriosis cells the former is pathogenetically a unique entity or, on the other hand, clinically that many carcinomas are diagnosed and treated in the early stages.

In the cases reported here, attack on the locoregional lymph nodes had already taken place; however, all 3 cases are still free of recurrences, in cases 2 and 3 even after almost 5 years which is rather in accord with a more favourable prognosis.

\section{Practical Conclusions}

In spite of its rare occurrence, the responsible physician should, in cases of peri- or postmenopausal patients with a history of endometriosis, when there is a clinical suspicion of an intestinal tumour in the lesser pelvis always take the possibility for a malignant transformation into consideration. Preoperative imaging procedures and biochemical tests are helpful for the interdisciplinary surgical planning but ultimately the suspected clinical and also intraoperative diagnosis can only be confirmed and managed on the basis of histological examinations. The objective is to encourage the pathologist to actively search for an atypical endometriosis and its malignant transformation and, in the case of women with a history of endometriosis and suspected intestinal adenocarcinoma, to employ the appropriate immunohistochemical examinations in the differential diagnosis. Exact knowledge of the origin is extremely important since a malignantly degenerated intestinal endometriosis requires different adjuvant therapy procedures than a primary intestinal carcinoma and apparently has a better stage-dependent prognosis.

\section{Conflict of Interest}

$\nabla$

None.

\section{References}

1 Sampson JA. Benign and malignant endometrial implants in the peritoneal cavity and their relation to certain ovarian tumors. Surg Gynec Obst 1924; 38: 287-311

2 Sampson JA. Endometrial carcinoma of the ovary, arising in endometrial tissue in that organ. Arch Surg 1925; 10: 1-72

3 Corner GW, Hu CY, Hertig AT. Ovarian carcinoma arising in endometriosis. Am J Obstet Gynecol 1950; 59: 760-774

4 Yantiss RK, Clement PB, Young R. Neoplastic and pre-neoplastic changes in gastrointestinal endometriosis: a study of 17 cases. Am J Surg Pathol 2000; 24: 513-524

5 Van Gorp T, Amant F, Neven P et al. Endometriosis and the development of malignant tumours of the pelvis. A review of the literature. Best Pract Res Clin Obstet Gynaecol 2004; 18: 349-371

6 Ulrich U, Wunschel A, Reichert VM et al. Endometrioseassoziierte maligne Tumoren. Gynäkologe 2015; 48: 221-227

7 Nasu K, Okamoto M, Kawano $Y$ et al. Endometrioid adenocarcinoma arising from intestinal endometriosis. JEPPD 2014; 6: 112-118

8 Erzen M, Rakar S, Klancnik B et al. Endometriosis-associated ovarian carcinoma (EAOC): an entity distinct from other ovarian carcinomas as suggested by a nested case-control study. Gynecol Oncol 2001; 83: $100-108$

9 Ulrich U, Hrsg. Interdisziplinäre S2k-Leitlinie für die Diagnostik und Therapie der Endometriose. München: Zuckschwerdt; 2013: 22

10 Kajihara H, Yamada Y, Shigetomi H et al. The dichotomy in the histogenesis of endometriosis-associated ovarian cancer: clear cell-type versus endometrioid-type adenocarcinoma. Int J Gynecol Pathol 2012; 31: 304-312

11 Swiersz ML. Role of endometriosis in cancer and tumor development. Ann N Y Acad Sci 2002; 955: 281-292

12 Ulrich $U$, Rhiem K, Richter $O$ et al. Entartungsrisiko und Assoziation mit malignen Erkrankungen. gynäkologie + geburtshilfe 2003; 5: 28-31

13 Modesitt SC, Tortolero-Luna G, Robinson JB et al. Ovarian and extraovarian endometriosis-associated cancer. Obstet Gynecol 2002; 100: 788795

14 Cuff J, Longacre TA. Endometriosis does not confirm improved prognosis in ovarian carcinoma of uniform cell type. Am J Surg Pathol 2012; 36: 688-695

15 Kumar S, Munkarah A, Arabi H et al. Prognostic analysis of ovarian cancer associated with endometriosis. Am J Obstet Gynecol 2011; 204: e61

16 Melin A, Lundholm C, Malki $N$ et al. Endometriosis as a prognostic factor for cancer survival. Int J Cancer 2011; 129: 948-955 